\title{
Diagnóstico de Hipertermia maligna: reporte de caso
}

\author{
Diagnostic of Malignant hyperthermia: a case presentation
}

\author{
Luz Enid Erazo Fino, ${ }^{1}$ Lastenia Antonia Chávez Villanueva, ${ }^{1}$ Lesly Mayela Torres Gonzáles ${ }^{2}$ \\ ${ }^{1}$ Médico General y Cirujano, Hospital General Santa Teresa, Comayagua, Secretaría de Salud Honduras. \\ ${ }^{2}$ Médico Especialista en Medicina Interna, Hospital General Santa Teresa, Comayagua, Secretaría de Salud Honduras.
}

\begin{abstract}
RESUMEN. Antecedentes: La Hipertermia maligna, desorden farmacogenético autosómico dominante, se presenta en pacientes susceptibles a gases anestésicos; estimándose predisposición genética en 1:3000 individuos generada por las variantes de los genes RYR1, CACNA1, STAC3, que explican la susceptibilidad del 77\%-86\% de la población caucásica europea. Se reporta incidencia entre 1:5000-100000 procedimientos de anestesia. Se induce por liberación excesiva sin recaptación adecuada de calcio citoplásmico desde el retículo sarcoplásmico, generando rigidez muscular, alto gasto de energía como ATP, hipermetabolismo, aumento secundario de $\mathrm{CO}_{2}$, calor, rabdomiólisis, hiperpotasemia, mioglobinuria y aumento de CPK. Descripción del caso clínico: Paciente masculino 18 años sometido a cirugía con anestesia general por apendicitis aguda, durante la cirugía presentó rigidez muscular, inestabilidad hemodinámica, hipertermia $\left(40^{\circ} \mathrm{C}\right)$, taquicardia (Frecuencia cardiaca: $120 \mathrm{lpm}$ ) e hipotensión (Presión Arterial 50/20), sin esfuerzo respiratorio y pérdida de reflejos osteotendinosos. Se diagnosticó hipertermia maligna y se inició manejo, sin embargo, paciente fallece poco menos de dos horas después de haber iniciado el cuadro. Conclusiones: La Hipertermia maligna, es una condición que genera un evento en respuesta a la exposición a agentes anestésicos durante una cirugía, cuadro difícil de prevenir. El manejo requiere celeridad y preparación exhaustiva que debe seguir las recomendaciones de manejo europeas y estadounidenses. Este es el primer caso reportado a nivel nacional y se acompaña con una revisión que permite determinar que, aunque no se disponga del fármaco clave en el sistema nacional para mejorar el pronóstico, la protocolización preventiva y terapéutica, así como el entrenamiento en simulación del personal de quirófano podrían influir en la supervivencia.
\end{abstract}

Palabras clave: Fiebre; Hipertermia maligna; Anestésicos; Periodo perioperatorio.

\section{INTRODUCCIÓN}

La hipertermia maligna (HM) es un desorden farmacogenético autosómico dominante que involucra mutación del receptor ryanodino 0 dihidropiridina. ${ }^{1,2}$ Esto induce a hipermetabolismo en el músculo esquelético en pacientes susceptibles a gases anestésicos como desflurano, isoflurano, sevoflurano, halotano y/o succinilcolina, aunque también son desencadenantes el golpe de calor, infecciones, estrés emocional, terapia con estatinas y el ejercicio extenuante. ${ }^{1,3}$ La patogenia consiste en liberación excesiva sin recaptación adecuada de calcio citoplásmico desde el retículo sarcoplásmico (RS), generando rigidez muscular persistente y alto gasto de energía como ATP con hipermetabolismo y aumento secundario de $\mathrm{CO}_{2}$, calor, rabdomiólisis, hiperpotasemia, mioglobinuria y aumento en la $\mathrm{CPK}^{3,4}$

La patología es dosis dependiente debido a la variabilidad interindividual, estimándose predisposición genética a HM en 1:3000 individuos generada por las variantes de los genes RYR1, CACNA1, STAC3, que explican la susceptibilidad de $77 \%-86 \%$ de la población caucásica europea ${ }^{4,5}$ (Ver cuadro 1) .

A pesar de estos progresos aún se desconocen muchos

Recibido: 22-3-2019 Aceptado para publicación 15-7-2019

Dirección para correspondencia: Luz Enid Erazo Fino

Correo electrónico: erazofino@yahoo.es

Conflictos de interés. Los autores expresan que no tienen conflictos de interés que declarar en relación a este artículo.

DOI: https://doi.org/10.5377//rmh.v87i2.11915 aspectos, ya que algunos casos requerirán en promedio tres exposiciones a anestésicos antes de presentar una crisis, lo que complica la determinación de la incidencia poblacional; la que se estima entre 1:10000-250000 personas ${ }^{2}$ no obstante, también se ha reportado incidencia entre 1:5000-100000 procedimientos de anestesia y aunque no se reportan diferencias étnicas, hay referencias sobre mayor riesgo entre franceses, escandinavos y japoneses así como en la población general de estados de Wisconsin, Nebraska, West Virginia y Michigan en EUA. ${ }^{1}$ Poco más de la mitad de los casos (52\%) se presentan en menores de 15 años y con mayor proporción en el sexo masculino (2:1) así como entre practicantes de fisicoculturismo, con media de edad de 18.3 años. ${ }^{3}$

Originalmente descrito en 1960 en un hombre joven con fractura de pierna que se presentó en el Hospital Royal Melbourne con historia familiar de fallecimientos relacionado con anestesia con cloruro de etilo. ${ }^{6}$ La hipertermia maligna es poco común, pero potencialmente letal y se presenta en el período perioperatorio, ${ }^{1,7}$ con el uso de dantrolene sódico y la mejora en los algoritmos de soporte, la mortalidad ha pasado de $64 \%$ $85 \%$ en 1980 a menos del $5 \%$ en la última década en los países desarrollados. ${ }^{3,7,8}$ Además de ser una patología rara, la presentación clínica es ampliamente variable, lo que la hace difícil de reconocer; algunos signos tempranos y específicos son aumento inexplicable del volumen tidal al final de la espiración de $\mathrm{CO}_{2}$ 
Cuadro 1. Clasificación de las mutaciones genéticas asociadas con la susceptibilidad para hipertermia maligna

\begin{tabular}{|c|c|c|}
\hline Mutación & Localización & Observaciones \\
\hline MSH 1 & Mutación asociada con el gen RYR1 en el locus cromosómico 19q13.1 & Mutación más frecuentemente descrita (> 50\%) \\
\hline MSH 2 & $\begin{array}{l}\text { Mutación asociada con el locus cromosómico 17q11.2-q24, relacionado con } \\
\text { el canal de sodio dependiente de voltaje del músculo esquelético. Posible } \\
\text { gen: SCN4A }\end{array}$ & Descrita en las familias norteamericanas y sudafricanas \\
\hline MSH 3 & $\begin{array}{l}\text { Mutación asociada con el locus cromosómico } 7 q 21-q 22 \text {, correspondiente al } \\
\text { sitio que codifica la subunidad ą2/ } \Delta \text { del receptor dihidropiridina, sensor del } \\
\text { voltaje del túbulo-T para el RyR. Posible gen: CACNL2A }\end{array}$ & Los genes causantes todavía no han sido ubicados \\
\hline MSH 4 & Mutación asociada con el locus cromosómico 3q13.1 & Los genes causantes todavía no han sido ubicados \\
\hline MSH 5 & $\begin{array}{l}\text { Mutación asociada con el gen codificador de la subunidad a1 del receptor de } \\
\text { dihidropiridina en el locus cromosómico 1q32. Gen CACLN1A3P }\end{array}$ & Presente en el $1 \%$ de los casos de hipertermia maligna \\
\hline MSH 6 & Mutación asociada con el locus cromosómico $5 p$ & La validez para la mutación MSH 6 tiene que ser confirmada \\
\hline
\end{tabular}

Fuente: Miller D. (2017).

$\left(\mathrm{ETCO}_{2}\right)$, aumento en el esfuerzo ventilatorio que es más rápido si se utilizó succinilcolina, además de asociarse con espasmo maseterino ${ }^{1,3} \mathrm{El}$ abrupto aumento en la temperatura que puede ser de $1-2^{\circ} \mathrm{C}$ cada 5 minutos a veces no es temprano, la rigidez muscular generalizada, taquicardia y disritmia pueden ayudar a definir la sospecha diagnóstica, evolucionando a hipermetabolismo con acidosis mixta fulminante descompensada, hiperpotasemia, elevación de la creatinkinasa y mioglobinuria; otros signos pueden ser piel marmórea, hipocalcemia y mioglobinuria (Ver cuadro 2). ${ }^{1,3}$ Se debe diferenciar de otras condiciones asociadas a la anestesia como distrofia muscular, miotonías, síndrome neuroléptico, sepsis, estrés quirúrgico, profundidad anestésica inadecuada, problemas de transfusión, Rigidez Muscular Maestra aislada (MMR), tirotoxicosis, feocromocitoma y sobrecalentamiento corporal iatrogénico; en niños hipovolemia, alergias, anticolinérgicos y alteraciones del SNC. 1,3,9

El manejo inicial consiste en sustituir el agente desencadenante, hiperventilación con oxígeno 100\% y abortar la cirugía. El dantrolene sódico es el antídoto farmacológico para HM, se administra de forma incremental en bolo IV a dosis inicial de 2.5 $\mathrm{mg} / \mathrm{kg}$ hasta $10 \mathrm{mg} / \mathrm{kg}$, aunque puede ser necesario administrar mayores dosis hasta estabilización cardíaca y respiratoria, con resolución o estabilización de la rigidez muscular. ${ }^{19,10} \mathrm{El}$ monitoreo anestésico debe continuar así como el de temperatura corporal, se debe implementar el protocolo de manejo para hipertermia, hiperkalemia, acidosis, oliguria, arritmias cardíacas, deben evitarse los bloqueadores de canales de calcio. ${ }^{1} \mathrm{Se}$ administran insulina (10U) más glucosa para la hiperkalemia y gluconato de calcio $(30 \mathrm{mg} / \mathrm{kg}$ ) además del tratamiento de soporte con traslado a unidad de cuidados intensivos (UCI); paralelamente al trabajo de control de la HM, se puede determinar el riesgo de $\mathrm{HM}$ aplicando la clasificación propuesta por Larach et al como sistema de puntuación ${ }^{11}$ y que se utiliza en EUA y Europa. Sin embargo, aún sin dantrolene, la implementación de un protocolo de manejo se relaciona con supervivencia, como el caso clínico reportado por Liu, Liu \& Wang. ${ }^{12}$

La identificación de la sospecha de riesgo de presentar HM en un paciente que será sometido a anestesia general asegura
Cuadro 2. Criterios para el diagnóstico de hipertermia maligna

\begin{tabular}{|c|c|}
\hline Hallazgo clínico & Manifestación \\
\hline Acidosis respiratoria & ET $\mathrm{CO}_{2}>55 \mathrm{mmHg} ; \mathrm{PaCO}_{2}>60 \mathrm{mmHg}$ \\
\hline Cardiaco & $\begin{array}{l}\text { Taquicardia sinusal inexplicable, taquicardia } \\
\text { ventricular o fibrilación ventricular }\end{array}$ \\
\hline Acidosis metabólica & Déficit de base $>8 \mathrm{~m} / \mathrm{EqL}$ o $\mathrm{pH}<7.25$ \\
\hline Rigidez muscular & $\begin{array}{l}\text { Rigidez generalizada; rigidez severa del músculo } \\
\text { masetero }\end{array}$ \\
\hline Degradación muscular & $\begin{array}{l}\text { Creatinkinasa sérica concentración }>20.000 \\
\text { unidades } / \mathrm{L} \text { Orina color obscuro Mioglobina } \\
\text { urinaria o sérica } \mathrm{K}+>6 \mathrm{mEq} / \mathrm{L}\end{array}$ \\
\hline Temperatura & Incremento rápido de la temperatura $>38.8^{\circ} \mathrm{C}$ \\
\hline Otros & $\begin{array}{l}\text { Reversión rápida de los signos de HM con la } \\
\text { administración de dantroleno, elevación de la } \\
\text { concentración de creatinkinasa en reposo }\end{array}$ \\
\hline Historia familiar & $\begin{array}{l}\text { Antecedentes por herencia autosómica } \\
\text { dominante }\end{array}$ \\
\hline
\end{tabular}

Fuente: Mullins M. (2018).

la supervivencia de este, esta sospecha se establece inicialmente con la historia familiar y antecedentes personales. Si se determina que podría haber riesgo de HM se recomienda realizar una prueba genética por el gen susceptible de HM, aunque el estándar de oro es la prueba de contractura muscular de cafeínahalotano que requiere de biopsia de músculo. ${ }^{1} \mathrm{Si}$ se determina riesgo de HM, se prepara con antelación la estación anestesiológica así como al personal y se planifican las acciones preventivas y de monitoreo, siguiendo las recomendaciones de la Asociación de Hipertermia Maligna de Estados Unidos de América (MHAUS) o del Grupo Europeo de Hipertermia Maligna. ${ }^{13}$

\section{PRESENTACIÓN DE CASO}

Paciente masculino de 18 años que ingresa a la sala de emergencias de un hospital de nivel secundario (departamental) por cuadro de apendicitis aguda; sin antecedente de medicación previa; al ingreso se indican Ampicilina 1 gramo IV c/6 
horas, Metronidazol $500 \mathrm{mg} \mathrm{IV} \mathrm{c/8horas} \mathrm{y} \mathrm{Gentamicina} 80 \mathrm{mg}$ IV c/12 horas. Paciente es sometido a cirugía con anestesia general una hora después del ingreso, se administró sevoflurano y succinilcolina y durante la cirugía presenta rigidez muscular, inestabilidad hemodinámica, hipertermia $\left(40^{\circ} \mathrm{C}\right)$, taquicardia (Frecuencia cardiaca: $120 \mathrm{lpm}$ ) e hipotensión (Presión arterial: $50 / 20$ ), sin esfuerzo respiratorio, pérdida de reflejos osteotendinosos, enrojecimiento generalizado. Se realizó interconsulta con medicina interna 10 minutos después de cirugía, se aplican medidas de soporte de emergencia con agentes vasoactivos (dobutamina, dopamina), control de temperatura (hielo local, compresas frías, alcohol), administración de suero salino IV, furosemida, bicarbonato y se solicitan pruebas de laboratorio para determinación de sodio, potasio, gases arteriales, CPK, $\mathrm{BUN}$, creatinina. Se inicia proceso de traslado a $\mathrm{UCl}$ a un hospital terciario, por lo crítico de su evolución se solicita de emergencia dantrolene a un hospital privado cercano, que envía de emergencia viales de $20 \mathrm{mg}$ y se inicia administración de este a dosis de $2.5 \mathrm{mg} / \mathrm{kg}$. Después de administrar la cuarta dosis, el paciente presenta paro cardiorespiratorio, aplicándose resucitación con éxito y continuándose la administración para suspenderse después de la novena dosis. Luego de la cual presenta un segundo evento de paro cardiorespiratorio con aplicación de resucitación sin éxito, declarándose fallecido poco menos de dos horas después de haber iniciado el cuadro. El manejo también incluyó Bicarbonato de Sodio, Insulina y medios físicos.

\section{DISCUSIÓN}

La HM secundaria a exposición a agentes anestésicos es una condición genética hereditaria de penetrancia variable que en parte de la población no es detectable ni con las pruebas de identificación genética disponibles. Se desconoce la incidencia real, ya que muchos casos pasan desapercibidos por presentar síntomas leves, por lo que incluso individuos expuestos a agentes anestésicos previamente y sin antecedentes de reacción a los mismos pueden presentar $\mathrm{HM}$, e incluso el $20 \%-25 \%$ presentará recrudescencia, por lo que se indica observación por 36-72 horas, el tiempo promedio es de 13 horas. ${ }^{5,14}$ Estas pruebas genéticas actualmente no están disponibles en Honduras, por lo que las opciones de prevención disponibles se limitan a la historia clínica personal y familiar para establecer la sospecha de riesgo de susceptibilidad genética de HM, lo que limita de forma importante la capacidad de respuesta, ya que las características de la instalación del cuadro no permiten dilaciones en la toma de decisiones y ejecución de acciones terapéuticas.

Aunque desde la introducción al mercado de dantrolene sódico para el tratamiento no sólo de HM, sino de otras condiciones similares en que hay rigidez muscular, la mortalidad ha disminuido de forma importante. Es un medicamento de difícil acceso, ya que sólo hay dos fabricantes, además de que su administración debe realizarse por personal capacitado y de forma temprana, ${ }^{3,10}$ así también se debe mantener un inventario de al menos 36 ampollas de $20 \mathrm{mg}$ que deben prepararse en 10-15 minutos de acuerdo a las guías de Manejo del Grupo Europeo de $\mathrm{HM},{ }^{15}$ recientemente fue introducida al mercado una nueva presentación de ampollas de $250 \mathrm{mg}$ (Ryanodex ${ }^{\mathrm{TM}}$ ); ${ }^{14}$ de alto costo y vida media muy corta. En el caso que se presenta en este trabajo, dantrolene se administra casi 90 minutos después del inicio del cuadro de HM por falta de disponibilidad inmediata en la institución, lo que podría haber influido en el pronóstico del paciente. Entre los principales obstáculos que dificultan y disminuyen la probabilidad de supervivencia está el tiempo de instalación de la sospecha diagnóstica desde el inicio de la reacción, que se ha revelado clave, esto sólo puede suceder en equipos quirúrgicos preparados con anticipación y entrenamiento de simulación de cuadros similares en pacientes con condiciones desconocidas, ya que la HM no es la única reacción de este tipo a agentes anestésicos.

El manejo inicia con la sospecha clínica temprana, como el cambio en el volumen final de espiración de $\mathrm{CO}_{2}$, contractura maseterina y rigidez muscular de cualquier intensidad, el aumento de temperatura puede presentarse 0 no de forma temprana y cuando sucede es de $1-2{ }^{\circ} \mathrm{C}$ cada 5 minutos..$^{1,3}$ Las pruebas iniciales de laboratorio no son útiles para el diagnóstico por la rapidez de la instalación del cuadro, por lo que se debe tener protocolizada la instalación de sospecha de HM y las acciones subsecuentes por parte del equipo de quirófano y de profesionales de enfermería para mantener la logística de mantenimiento de recursos disponibles que, aunque existan en la institución, no podrían estar disponibles con la premura que el cuadro exige.

Otros autores han reportado ya casos de supervivencia pediátrica aplicando medidas protocolizadas con acciones en tiempo y forma, que han resultado en supervivencia sin complicaciones en hospitales que no disponían de dantrolene, demostrándose que el tratamiento de soporte juega un rol esencial en la supervivencia. ${ }^{9,12}$ Entre las medidas de prevención que algunos autores reportan como eficaces en medios como el nuestro, en que no hay medios de identificación temprana precisos, están el monitoreo permanente de temperatura corporal durante la cirugía. En una publicación por Gleich et al se describe un instrumento útil para detección temprana perioperatoria de HM, aunque aún se necesita más evidencia para concluir sobre su utilidad, parece promisorio ${ }^{3,16}$

La HM es reconocida como una condición que puede generar un evento en respuesta a la exposición de agentes anestésicos durante una cirugía y que es difícil de prevenir, aún en países industrializados. El manejo de estos casos requiere de celeridad y preparación exhaustiva que debe seguir las recomendaciones de manejo europeas y estadounidenses. Este es el primer caso reportado a nivel nacional, con una revisión que permite determinar que, aunque no se disponga del fármaco clave en el sistema nacional para mejorar el pronóstico de estos casos, la protocolización preventiva y terapéutica, así como el entrenamiento en simulación del personal de quirófano podrían influir en la supervivencia de estos pacientes. 


\section{REFERENCIAS}

1. Mullins MF. Malignant hyperthermia: a review. J Perianesth Nurs. 2018;33(5):582-9.

2. Litman RS, Griggs SM, Dowling JJ, Riazi S. Malignant hyperthermia susceptibility and related diseases. Anesthesiology. 2018;128(1):159-67.

3. Smith JL, Tranovich MA, Ebraheim NA. A comprehensive review of malignant hyperthermia: preventing further fatalities in orthopedic surgery. J Orthop. 2018;15(2):578-80.

4. Kraeva N, Sapa A, Dowling JJ, Riazi S. Malignant hyperthermia susceptibility in patients with exertional rhabdomyolysis: a retrospective cohort study and updated systematic review. Can J Anaesth. 2017;64(7):736-43.

5. Miller DM, Daly C, Aboelsaod EM, Gardner L, Hobson SJ, Riasat K, et al. Genetic epidemiology of malignant hyperthermia in the UK. Br J Anaesth. 2018;121(4):944-52.

6. Schiemann AH, Bjorksten AR, Gillies RL, Hockey BM, Ball C, Pollock N, et al. A genetic mystery in malignant hyperthermia 'solved'? Br J Anaesth. 2018;121(3):681-2.

7. In J, Ahn EJ, Lee DK, Kang H. Incidence of malignant hyperthermia in patients undergoing general anesthesia: protocol for a systematic review and meta-analysis. Medicine. 2017;96(49):e9115.

8. Sinha AK, Kumari P, Vaghela MM, Sinha C, Kumar B. Postoperative malignant hyperthermia- a medical emergency: a case report and review of literature. J Clin Diagn Res. 2017;11(4):PD01-PD2.

9. Glahn KP, Ellis FR, Halsall PJ, Muller CR, Snoeck MM, Urwyler A, et al. Recognizing and managing a malignant hyperthermia crisis: guide- lines from the European Malignant Hyperthermia Group. $\mathrm{Br} \mathrm{J}$ Anaesth. 2010;105(4):417-20.

10. Riazi S, Kraeva N, Hopkins PM. Malignant hyperthermia in the postgenomics era: new perspectives on an old concept. Anesthesiology. 2018;128(1):168-80

11. Larach MG, Localio AR, Allen GC, Denborough MA, Ellis FR, Gronert GA, et al. A clinical grading scale to predict malignant hyperthermia susceptibility. Anesthesiology. 1994;80(4):771-9.

12. Liu ST, Liu LF, Wang SY. Treatment of malignant hyperthermia without dantrolene in a 14-year-old boy. Chin Med J. 2017;130(6):755-6.

13. Neira VM, Al Madhoun W, Ghaffari K, Barrowman N, Berrigan P, Splinter W. Efficacy of malignant hyperthermia association of the United States-Recommended methods of preparation for malignant myperthermia-susceptible patients using drager zeus anesthesia workstations and associated costs. Anesth Analg. 2019; 129(1):74-83.

14. Riazi S, Kraeva N, Hopkins PM. Updated guide for the management of malignant hyperthermia. Can J Anesth. 2018;65(6):709-21.

15. Kim HJ, Koh WU, Choi JM, Ro YJ, Yang HS. Malignant hyperthermia and dantrolene sodium. Korean J Anesthesiol. 2019; 72(1):78-9.

16. Gleich SJ, Strupp K, Wilder RT, Kor DJ, Flick R. An automated real-time method for the detection of patients at risk for malignant hyperthermia. Paediatr Anaesth. 2016;26(9):876-82.

ABSTRACT. Background: Malignant hyperthermia is an autosomal dominant pharmacogenetic disorder that occurs in patients susceptible to anesthetic gases; estimating genetic predisposition in 1:3000 individuals generated by the variants of genes RYR1, CACNA1, STAC3, which explain the susceptibility of $77 \%-86 \%$ of the caucasian population; incidence is reported 1:5000-100,000 anesthesia procedures. It is induced by excessive release without adequate reuptake of cytoplasmic calcium from the sarcoplasmic reticulum, generating persistent muscle stiffness and high energy expenditure such as ATP, hypermetabolism and secondary increase in CO2, heat, rabomyolysis, hyperkalemia, myoglobinuria and increase CPK. Clinical case description: Patient 18-year-old male, underwent surgery with general anesthesia due to acute appendicitis, during the surgery presented muscle rigidity, hemodynamic instability, hyperthermia $\left(40^{\circ} \mathrm{C}\right.$ ), tachycardia $120 x^{\prime}$ and hypotension (Blood presure 50/20), without respiratory effort and loss of osteotendinous reflexes. Diagnosis of malignant hyperthermia and management is started; however, the patient dies a little less than two hours after starting the syndrome. Conclusions: Malignant hyperthermia is a condition that can generate an event in response to the exposure of anesthetic agents during a surgery, and it is difficult to prevent. The handling of these cases requires speed and thorough preparation that must follow the European and American management recommendations. This is the first case reported at national level, with a review that allows to determine that although the key drug in the national system is not available to improve the prognosis of these cases, the preventive and therapeutic protocolization, as well as the simulation training of the personnel of surgery could influence the survival of these patients.

Keywords: Fever; Malignant hyperthermia; Anesthetics; Perioperative period. 